\title{
A cellular automaton model for tumour growth in inhomogeneous environment
}

\author{
T. Alarcón ${ }^{\mathrm{a}, *}$, H.M. Byrne ${ }^{\mathrm{b}}$, P.K. Maini ${ }^{\mathrm{a}}$ \\ ${ }^{a}$ Centre for Mathematical Biology, Mathematical Institute, University of Oxford, 24-29 St Giles', Oxford OX1 3LB, UK \\ ${ }^{\mathrm{b}}$ Centre for Mathematical Medicine, Division of Applied Mathematics, School of Mathematical Sciences, \\ University of Nottingham, Nottingham NG7 2RD, UK
}

Received 16 October 2002; received in revised form 30 May 2003; accepted 11 June 2003

\begin{abstract}
Most of the existing mathematical models for tumour growth and tumour-induced angiogenesis neglect blood flow. This is an important factor on which both nutrient and metabolite supply depend. In this paper we aim to address this shortcoming by developing a mathematical model which shows how blood flow and red blood cell heterogeneity influence the growth of systems of normal and cancerous cells. The model is developed in two stages. First we determine the distribution of oxygen in a native vascular network, incorporating into our model features of blood flow and vascular dynamics such as structural adaptation, complex rheology and red blood cell circulation. Once we have calculated the oxygen distribution, we then study the dynamics of a colony of normal and cancerous cells, placed in such a heterogeneous environment. During this second stage, we assume that the vascular network does not evolve and is independent of the dynamics of the surrounding tissue. The cells are considered as elements of a cellular automaton, whose evolution rules are inspired by the different behaviour of normal and cancer cells. Our aim is to show that blood flow and red blood cell heterogeneity play major roles in the development of such colonies, even when the red blood cells are flowing through the vasculature of normal, healthy tissue.
\end{abstract}

(C) 2003 Elsevier Ltd. All rights reserved.

Keywords: Tumour growth; Blood flow; Heterogeneity; Cellular automaton

\section{Introduction}

Solid tumour growth has attracted the attention of theoreticians from many different fields, becoming one of the most important areas of active research in the theoretical biology community. The number of models analysing the different stages in tumour development, from its initial avascular phase to invasion and metastasis through vascularization via tumour-induced angiogenesis, is huge. Nevertheless, almost all of these models neglect a factor which can, eventually, influence many features of the growth process. The factor in question is that tumour growth actually occurs in a heterogeneous environment (Baum et al., 1999). Our aim in this paper is to study, by means of a cellular automaton model, two different processes: the growth of a heterogeneous colony composed of two, competitive cell populations and the initial stages of tumour invasion. We will investigate the

\footnotetext{
*Corresponding author. Tel.: + 1865280610; fax: +1865270515 .

E-mail address: alarcon@maths.ox.ac.uk (T. Alarcón).
}

effect on each of these processes of the heterogeneity of the supporting vasculature and the rate of oxygen delivery to the cell populations.

Although many factors contribute to the heterogeneity of the environment in which our cells proliferate, we focus our attention on blood flow. Despite the highly organized structure of vasculature in normal tissue, as compared to the chaotic appearance of vascular beds in cancer tissue (Baish et al., 1996), the blood flow distribution in normal tissue is, for a number of reason, strongly inhomogeneous. Firstly, blood is a complex suspension of different elements, with a complex rheology (Pries et al., 1994). Secondly, the vascular system is not a network of rigid tubes but a structure which interacts with the flow and remodels itself accordingly (Pries et al., 1998). Additionally, haematocrit, i.e. the fraction of the total volume of blood occupied by red blood cells, is not distributed at bifurcations in the same way as blood flow (Fung, 1993). Consequently, the distribution of oxygen in the vascular network is highly irregular. 
Our aim in this paper is to study how the phenomena mentioned above affect the growth of cellular colonies and malignant invasion of healthy tissue. To this end our procedure will be as follows. The first step is to simulate blood and haematocrit (i.e. oxygen) flow through a regular network of primitive vasculature. We take into account real features of blood rheology as well as adaptation of the vascular network via a remodelling algorithm. This enables us to determine the haematocrit distribution. The next step is to couple this with a hybrid cellular automaton model for the tissue dynamics of colony growth and tumour invasion.

Although our simulated environment is still a highly idealized version of the situation in vivo, it is a more realistic description than the usual assumption of tissue homogeneity. Accordingly, we hope that our model will produce more realistic results than existing models and provide a framework that can be further developed.

Cellular automata have been used extensively to model a wide range of problems (Wolfram, 1986; Ermentrout and Edelstein-Keshet, 1993). Biological systems are particularly suitable for analysing by cellular automata (Ermentrout and Edelstein-Keshet, 1993). In particular, cellular automata models have been used to model many aspects of tumour growth and therapy (Duchting and Vogelsaenger, 1985) and the presence of immune surveillance (Qi et al., 1993). More recently, Kansal et al. (2000) have developed a cellular automaton for avascular tumour growth on a Voronoi lattice. The use of this type of lattice preserves the discrete nature of cells but removes the anisotropy introduced by the use of a regular lattice. Patel et al. (2001) have developed a cellular automaton model to study the role of acidity in tumour growth. Tumour-induced angiogenesis has also been modelled using a cellular automaton approach (Anderson and Chaplain, 1998).

The paper is organized as follows. In Section 2, we explain how we construct a heterogeneous environment from blood flow simulations in a network of native vessels. Section 3 deals with cell dynamics in this inhomogeneous environment. In this section, we describe in detail our cellular automaton model and explain how these dynamics are affected by the heterogeneity of the environment. In Sections 4 and 5 we consider two different situations: in Section 4 we consider the growth of a colony of both normal and cancerous cells; in Section 5 we consider the invasion of healthy tissue by a malignant colony of cells. Finally, in Section 6, we discuss our results and present our conclusions.

\section{Adaptation of the vascular network}

As mentioned in the Introduction, our aim is to study the growth of cell colonies in heterogeneous environments, similar to those found in vivo. Of particular interest here is the impact on colony growth of the inhomogeneous distribution of oxygen that is produced by blood flow heterogeneity.

To produce realistic results, we have two options: we can either seek experimental data on vascular bed morphology and hydrodynamic parameters or we can simulate such an environment, incorporating generic, but realistic, features of the vascular system. In the present work we have chosen the latter approach. Our procedure is to implement an adaptation mechanism which incorporates a number of characteristics that have been observed experimentally and generates a generic structure whose morphology depends on hydrodynamics parameters. This does not reproduce any concrete example of a vascular networks, as would be the case if we were using real experimental data. However it produces a network exhibiting features that are typical of the vascular system.

The adaptation mechanism we use relates to experimental observations by Pries et al. (1995) of real haemodynamic features. The vascular system is believed to be organized to comply with a number of physical design principles so as to optimise some related function (LaBarbera, 1990). The first design principle was developed by Murray (1926). He hypothesized that the vascular tree is organized to minimize its energetic costs, these costs including the power needed to sustain the blood flow and the metabolic energy necessary to make and maintain blood. By minimizing the associated function, Murray concluded that, in an optimal vessel, the flow rate is proportional to the cube of the lumen radius (Murray's law). A consequence of Murray's law is that the wall shear stress is uniform throughout the network (Zamir, 1977). It is widely known that wall shear stress influences smooth muscle tone and vessel wall structure. In consequence we deduce that the vascular tree must autoregulate itself to maintain the wall shear stress at its fixed value.

Although fairly constant values of the wall shear stress have been reported for arteries and large arterioles, lower values are reported for the microcirculation, venules and veins. This raises questions about the general applicability of Murray's hypothesis. Another factor to which blood vessels are exposed is transmural pressure, which, like shear stress, affects vessel diameter both acutely and chronically. Consequently, transmural pressure is likely to alter shear-dependent responses of the vascular network. Pries and co-workers studied the hydrodynamic design of a terminal vascular bed in an attempt to explain how the coupling between transmural pressure and wall shear stress is accomplished (Pries et al., 1995). They found that the wall shear stress exhibits a sigmoidal shape which seems to be universal for arterioles, capillaries and venules, contradicting Murray's law (see Pries et al., 1995; Alarcón et al., 
2002). We have recently proposed an extension of Murray's design principle, which accounts for this dependence of the wall shear stress on the transmural pressure (Alarcón et al., 2002). We minimize Murray's energy dissipation function, but we consider the actual dependence of blood relative viscosity upon the radius of the vessel. We show that a branching network of vessels constructed according to the corresponding design principle reproduces the sigmoidal shape of the wall shear stress. We also show that our results can be extended to compliant vessels.

\subsection{Structural adaptation}

Based on the above information about the hydrodynamics of vascular beds and on the fact that the vascular system continually adapts to the demands of the surrounding tissue, ${ }^{1}$ Pries et al., formulated an adaptation mechanism which describes how the lumen radius, $R(t)$, is modified by these effects (Pries et al., 1998):

$$
\begin{aligned}
R(t+\Delta t)= & R(t)+R \Delta t\left(\log \left(\frac{\tau_{w}}{\tau(P)}\right)\right. \\
& \left.+k_{m} \log \left(\frac{\dot{Q}_{r e f}}{\dot{Q} H}+1\right)-k_{s}\right) .
\end{aligned}
$$

In Eq. (1) $\Delta t$ is the time-scale, $\dot{Q}$ is the flow rate, $\dot{Q}_{\text {ref }}$, $k_{m}$ and $k_{s}$ are constants described below, $H$ is the haematocrit, $\tau_{w}=R \Delta P / L$ is the wall shear stress acting on a vessel of length $L$. For Poiseuille flow, $\tau_{w}=4 \mu \dot{Q} / \pi R^{3} . P$ is the transmural pressure, $\Delta P$ is the change in $P$ along $L$, and $\tau(P)$ the magnitude of the associated wall shear stress. In Pries et al. (1998) a third stimulus, the so-called conducted stimulus, is introduced which, for simplicity, we neglect in our simulations, as Eq. (1) provides the simplest stable adaptation mechanism. The expression for $\tau(P)$ that we use was obtained by fitting to data from the rat mesentery, and is given by Pries et al. (1998):

$$
\tau(P)=100-86[\exp (-5000 \log (\log P))]^{5.4} \text {. }
$$

The second term on the right-hand side of Eq. (1), i.e. the difference between the logarithms of the wall shear stress and its corresponding set point value, represents the response to mechanical or hemodynamic stimuli. It is proposed (Pries et al., 1995) that vascular networks adapt themselves in order to maintain a fixed relationship (given in Eq. (2)) between transmural pressure and wall shear stress. This feature is implemented via this term in Eq. (1). Equally, structural adaptation of vascular beds also occurs in response to the metabolic demands of the surrounding tissue. Consequently, if the

\footnotetext{
${ }^{1}$ This implies that network morphology is not only determined by genetic information but also by physical mechanisms that act locally in response to mechanical, metabolic and biochemical stimuli.
}

flow in some vessels drops and the tissue becomes poorly supplied with oxygen or other metabolites, then the vasculature will be stimulated to grow. This phenomenon is accounted for by the third term in the right-hand side of Eq. (1). We remark that the metabolic stimulus is always positive and increases with decreasing red cell flux, $\dot{Q} H$. The constant $k_{m}$ indicates how rapidly the vasculature adapts to the metabolic needs of the tissue. The constant $k_{s}$ represents the so-called shrinking tendency and accounts for the need for growth factors to maintain or increase the size of a given vessel.

\subsection{Introduction of blood rheology and haematocrit}

In Pries et al. (1998), blood viscosity was assumed to be constant. In our simulations we include the effects of complex blood rheology and haematocrit in the adaptation mechanism.

\subsubsection{Blood rheology}

Blood is far from being a simple fluid with constant viscosity: it is a complex suspension of cells and molecules of a wide range of sizes. Thus, modelling blood as a Newtonian fluid is a very crude approximation. Here we are not going to examine the problem of blood rheology in all its complexity. Instead, we focus on the effects of red blood cells, as they seem to play a major role in blood flow (Fung, 1993). As a result, we assume, henceforth, that blood is a suspension of red blood cells in a Newtonian fluid. As we shall see, even for this simplified situation, the behaviour of the blood viscosity is non-trivial.

Let us assume that blood is flowing through a tube of radius $R$ and that the volume fraction occupied by the red blood cells (i.e. the haematocrit) is $H$. For a given $H$, the viscosity of the blood depends nonmonotonically on $R$ and three different flow regimes may be identified. If $R$ is much greater than the typical size of a red blood cell, ${ }^{2}$ the viscosity is independent of $R$. As $R$ decreases the viscosity also decreases (the Fahraeus-Lindqvist effect). This behaviour persists until the vessel radius is of the order of $15-20 \mu \mathrm{m}$. The viscosity then reaches a minimum and, thereafter, increases if $R$ is similar in magnitude to the radius of a red blood cell (Pries et al., 1994).

The reasons for this dependence of the viscosity on the vessel radius for large and small values of $R$ are quite clear. However, the physical mechanism leading to the Fahraeus-Lindqvist effect is less obvious. An explanation may be found by considering the behaviour of the haematocrit itself when blood flows from a large reservoir (or a tube of large radius) into a small tube. In such situations, the haematocrit diminishes: the smaller the tube the smaller is the haematocrit. Moreover, when

\footnotetext{
${ }^{2}$ Average red blood cell diameter in humans is $7-8 \mu \mathrm{m}$.
} 


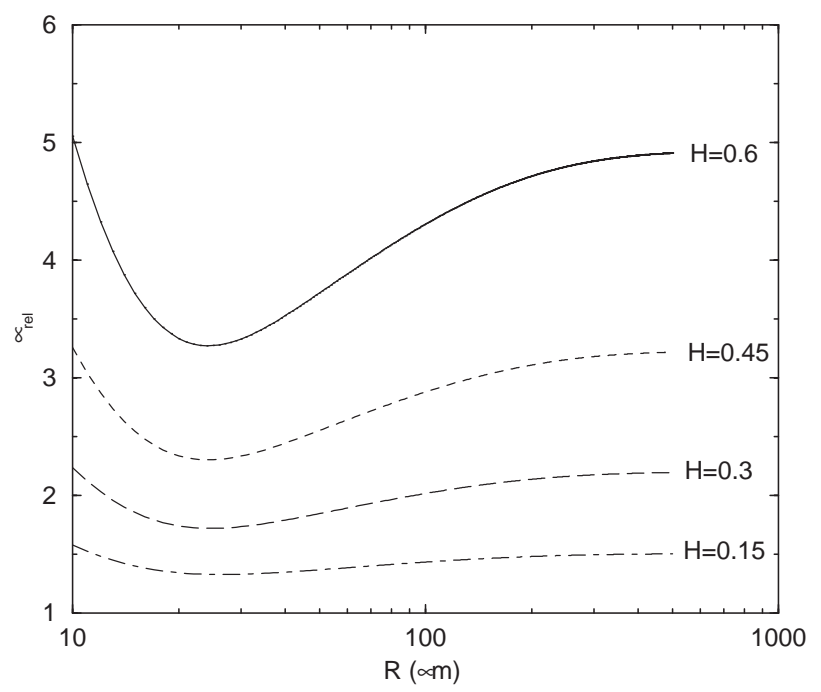

Fig. 1. Graph showing how the relative viscosity $\mu_{r e l}$ varies with the vessel radius $R$ for fixed values of the haematocrit $H$ (see Eqs. (3)).

a suspension of red blood cells flows through a tube, the cells tend to accumulate in the centre of the tube, far from the walls, thereby reducing the friction force that they experience. The dependence of blood viscosity on the haematocrit for a given radius is monotonically increasing with $H$ (Fung, 1993).

To the best of our knowledge, a theoretical model that captures all the above features of blood viscosity over a wide range of radii and haematocrit does not currently exist. However, Pries et al. managed to fit the following explicit expression for the viscosity as a function of $R$ and $H$ to detailed experimental data (Pries et al., 1994):

$$
\begin{aligned}
\mu_{\text {rel }}= & {\left[1+\left(\mu_{0.45}^{*}-1\right) \frac{(1-H)^{C}-1}{(1-0.45)^{C}-1}\left(\frac{2 R}{2 R-1.1}\right)^{2}\right] } \\
& \left(\frac{2 R}{2 R-1.1}\right)^{2}, \\
\mu_{0.45}^{*}= & 6 \mathrm{e}^{-0.17 R}+3.2-2.44 \mathrm{e}^{-0.06(2 R)^{0.645}}, \\
C= & \left(0.8+\mathrm{e}^{-0.15 R}\right)\left(-1+\frac{1}{1+10^{-11}(2 R)^{12}}\right) \\
& +\frac{1}{1+10^{-11}(2 R)^{12}} .
\end{aligned}
$$

In Eqs. (3) $\mu_{r e l}$ is the real viscosity divided by the viscosity of the plasma. The behaviour of $\mu_{r e l}$ as a function of $R$ for different values of $H$ is shown in Fig. 1.

The features displayed by the viscosity give rise to a highly complex, non-linear problem, in which the viscosity depends on the dynamical state of the entire vascular network, as the viscosity depends on the radius. The vessel radii, in turn, depend on the hydrodynamic quantities through the adaptation mechanism, and the

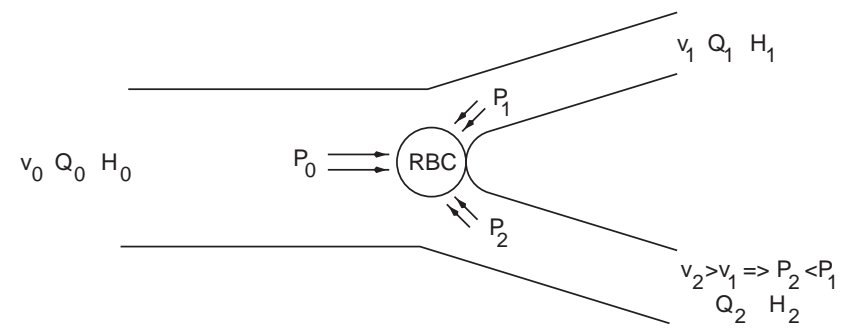

Fig. 2. Schematic representation of a vessel bifurcation and the mechanism that gives rise to uneven haematocrit distribution at bifurcations. RBC stands for red blood cell. See text for details.

hydrodynamic state of each vessel depends on the state of the entire network.

\subsubsection{Computation of the haematocrit}

Since $\mu_{r e l}$ depends on $H$, the haematocrit distribution is an important factor when determining the hydrodynamic state of the network. The simplest way to proceed is to assume that at each bifurcation the distribution of $H$ depends on the flow velocity in each of the daughter vessels (Fung, 1993). Roughly speaking, a larger proportion of the haematocrit from the parent vessel is transported along the faster branch. Additionally, it has been observed that at bifurcations where the ratio between the velocities of the branches exceeds a certain threshold, all the haematocrit enters the faster branch. Combining these results, we assume that, at a bifurcation, the haematocrit is computed as follows ${ }^{3}$ (see Fig. 2 for definitions):

$H_{p}=H_{1}+H_{2}$,

$\frac{H_{1}}{H_{2}}=\alpha \frac{v_{1}}{v_{2}}$, if $\frac{v_{1}}{v_{2}}<T H R$,

$H_{1}=H_{p}$, if $\frac{v_{1}}{v_{2}}>T H R$.

In Eq. (4) $v_{i} \equiv \dot{Q}_{i} / \pi R_{i}^{2}$ is the average flow velocity on a section orthogonal to the axis of the vessel, $\alpha$ is a phenomenological parameter which accounts for strength of the non-symmetry of the haematocrit distribution at bifurcations, and $T H R$ is the value of the ratio between the velocities of the branches above which all the haematocrit goes to the faster branch.

Taking all these factors into account, our algorithm for the structural adaptation of a vascular bed is as follows:

1. The total flow rate through the network is determined by the pressure drop between two points of the

\footnotetext{
${ }^{3}$ Although supported by experimental evidence, Eq. (4) is not entirely satisfactory from the theoretical point of view, as it is not symmetric, meaning that we need to know a priori which branch is faster. However, this is always possible to know in our simulations, so this fact does not affect our results. A more elaborated law for haematocrit splitting at bifurcations will be developed in future extensions of the present work.
} 


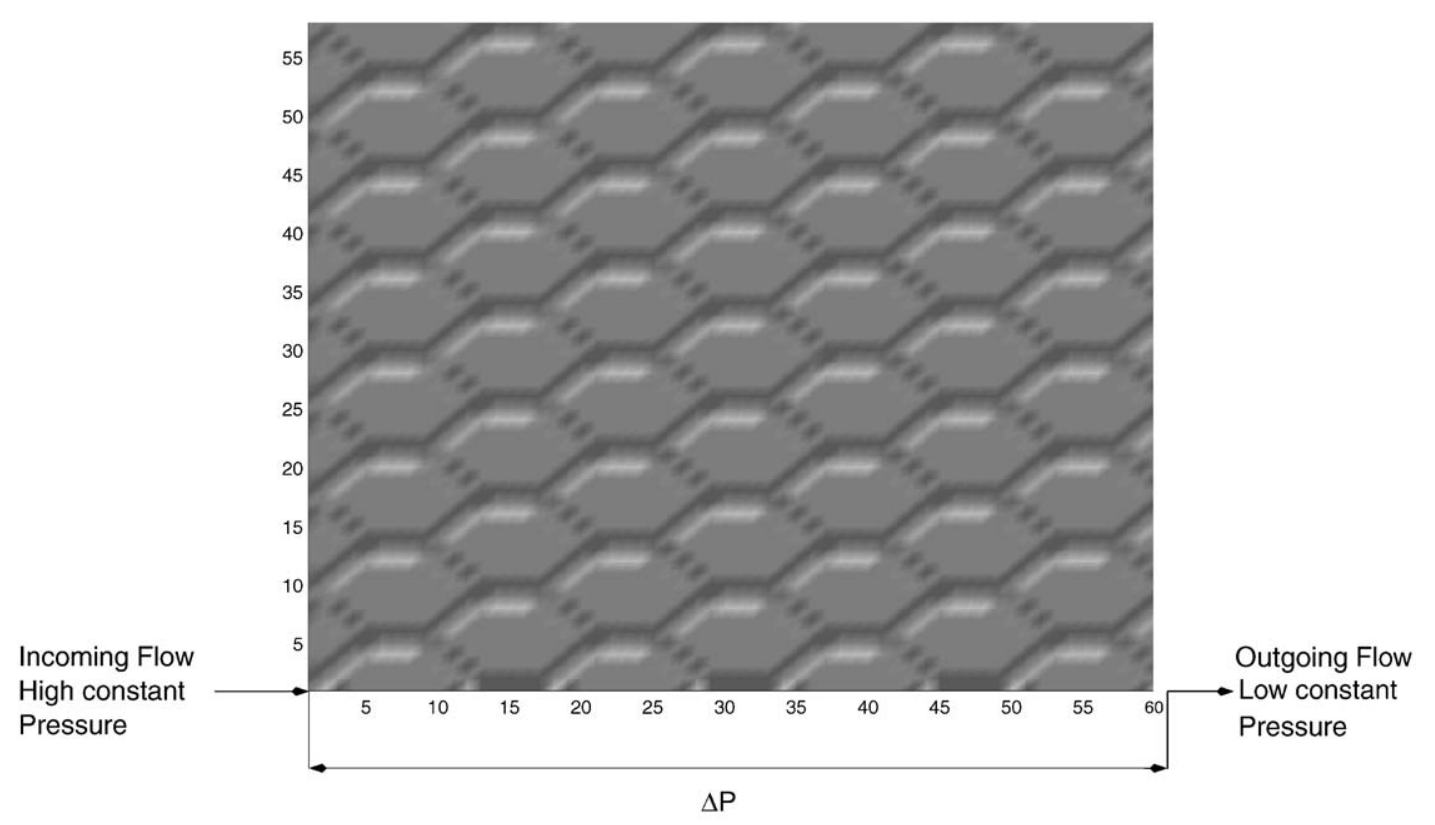

Fig. 3. Initial condition for the adaptation algorithm. All the vessels have the same initial radius $(10 \mu \mathrm{m})$ and length $(80 \mu \mathrm{m})$.

network as shown in Fig. 3 (corresponding to the arterial and venous sides). An initial haematocrit, $H_{T}$ is also prescribed. The flow in each vessel is assumed to be laminar steady Poiseuille's flow, i.e.

$$
\begin{aligned}
& \Delta P=\dot{Q} Z, \\
& Z=\frac{8 \mu(R) L}{\pi R^{4}},
\end{aligned}
$$

where $\dot{Q}$ is the flow rate in each vessel, and $Z$ its resistance. In practice, our simulations yield the current in each vessel, $J$, which is related to the flow rate by $\dot{Q}=|J|$.

2. Given the initial network configuration (i.e. radii, lengths and viscosity of all the vessels), we compute the flow rates through, and pressure drops across, each vessel using Kirchoff's laws. This allows us to calculate the pressure in each vessel, relative to a constant reference pressure (i.e. the pressure on the arterial or venous side). The wall shear stress, $\tau_{w}=$ $R \Delta P / L$, is computed in terms of the flow rate and resistance in each vessel.

3. The distribution of haematocrit is computed using Eq. (4).

4. The radius of each vessel is updated using Eq. (1).

5. Once we have updated the haematocrit and radii, the viscosity of each vessel is computed. The new state of the network is fed back into step 2 and the corresponding flow pattern computed.

6. Steps $2-5$ are repeated until a stationary state is reached.

This adaptation algorithm has been applied to a hexagonal vascular network (see Fig. 3), similar to that observed in the avian yolk sac (Honda and Yoshizato,
1997). Initially, all the vessels have the same lumen diameter and the same length. As the distribution of flow rates is inhomogeneous (for example, the flow rates near to the inlet and outlet points are bigger than in the rest of the network), the corresponding values of the wall shear stress will differ throughout the network. Accordingly, from Eq. (1), we deduce that the values of the different adaptation stimuli will vary across the vascular bed. This implies that our structural adaptation algorithm generates an inhomogeneous distribution of radii from an initially uniform network. In addition, since the viscosity depends on the haematocrit and the haematocrit depends on the flow rates (see Eq. (4)), there is a feed-back between the flow rate, haematocrit distribution, and radius distribution.

Typical results for the stationary current and haematocrit patterns are shown in Fig. 4. Comparison between the upper and lower plots in Fig. 4 reveals that the haematocrit is distributed more unevenly than the flow rate. This is a consequence of Eq. (4), which implies that, at bifurcation points, more red blood cells enter branches with high flow rates. Blood flow also exhibits a high degree of heterogeinity, in agreement with recent results reported by McDougall et al. (2002).

\section{Cell dynamics}

Having determined the haematocrit (i.e. oxygen distribution) in a vascular network, we now proceed to investigate how a colony of cells evolves in response to the associated distribution of oxygen in the tissue.

We begin by locating a small heterogeneous colony in the space between the vasculature. The colony is 

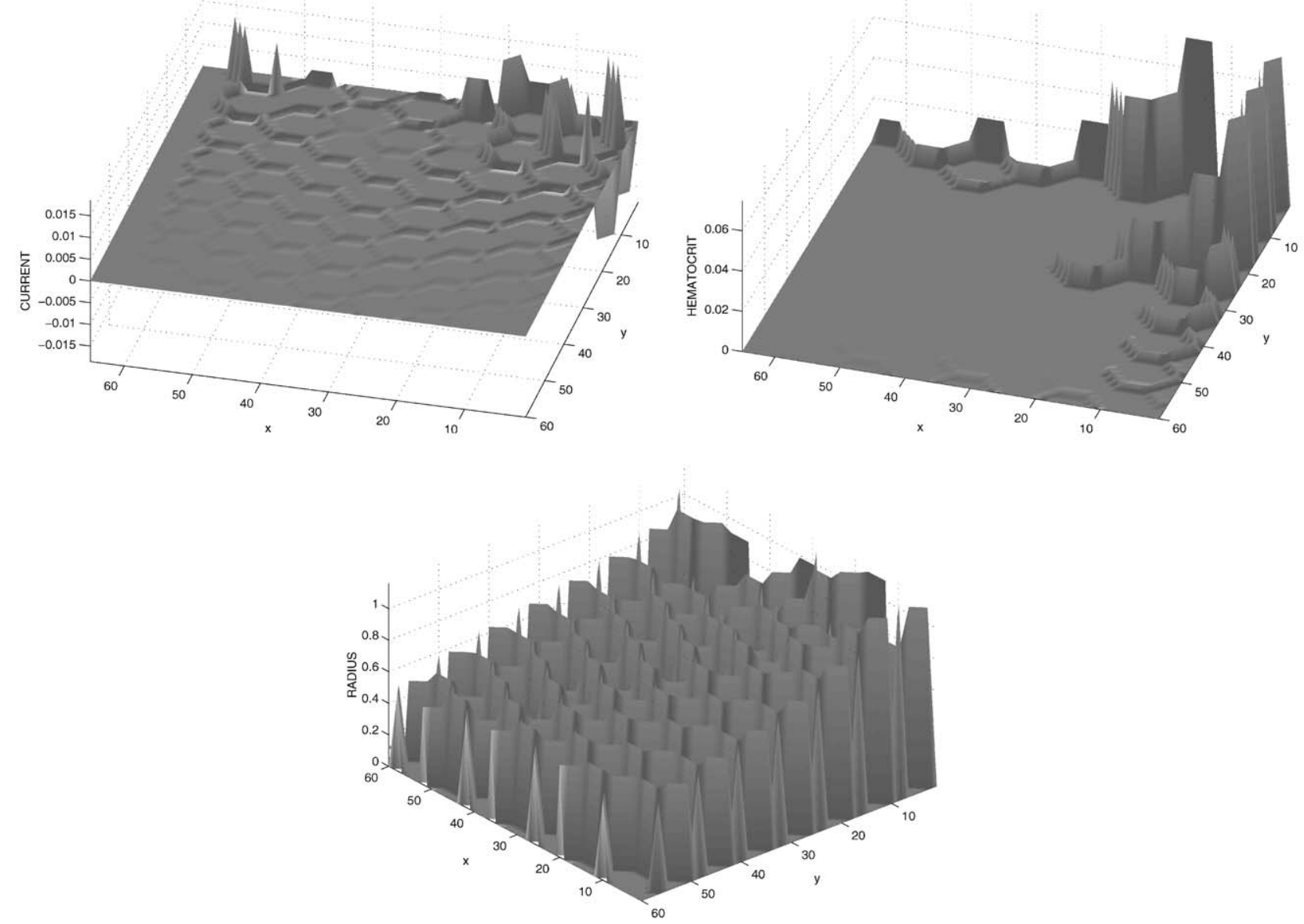

Fig. 4. Current (upper figure), haematocrit, and radius (bottom figure) patterns after 500 iterations of the remodelling algorithm described in the text. Parameters given in Table 1.

composed of normal and cancerous cells and its evolution is modelled using a hybrid cellular automaton (we call the automaton 'hybrid' since, as we explain below, it combines discrete and continuous fields). This approach was used to determine the effects of acidity on tumour growth (Patel et al., 2001).

Our two-dimensional model consists of an array of $N \times N$ automaton elements, which will eventually be identified with real cells. The state of each element is defined by a state vector, whose components correspond to features of interest. Here, the state vector has three components: (i) occupation, i.e. whether an element is occupied by a normal cell, a cancer cell, an empty space or a vessel, (ii) cell status, i.e. whether the cell is in a proliferative or a quiescent state, and (iii) the local oxygen concentration. The state vector evolves according to prescribed local rules which determine the state of a given element from its own state and that of its neighbours' on the previous time step. In the present case, we consider a simple square lattice, i.e. each cell has four (nearest) neighbours.

For simplicity we assume that the vasculature does not evolve, i.e. we neglect the effects of angiogenesis and vessel regression. All spaces between the vessels are empty except for those occupied by the initial colony. The colony initially occupies a $10 \times 10$ (in automaton element units) region in the centre of the array. ${ }^{4} \mathrm{An}$ (empty) element in this initial colony is occupied with probability $1 / 2$ by a normal or cancer cell (see Fig 5). In our model there is a one-to-one correspondence between automaton elements and real cells, the size of an element $(\Delta \times \Delta)$ corresponding approximately to the size of a typical cell, i.e. $\Delta \sim 20 \mu \mathrm{m}$.

While in our model the cells are viewed as discrete entities, the oxygen concentration is treated as a continuous field, as the typical length of a molecule is very small compared to the characteristic size of a cell. The time evolution of the oxygen concentration is governed by a partial differential equation (PDE), with sinks, sources and boundary conditions determined by the corresponding distribution of cells and vessels (see Eq. (7) in Section 3.2).

\footnotetext{
${ }^{4}$ The upper left corner of the colony is placed at the element in the position $(20,20)$.
} 


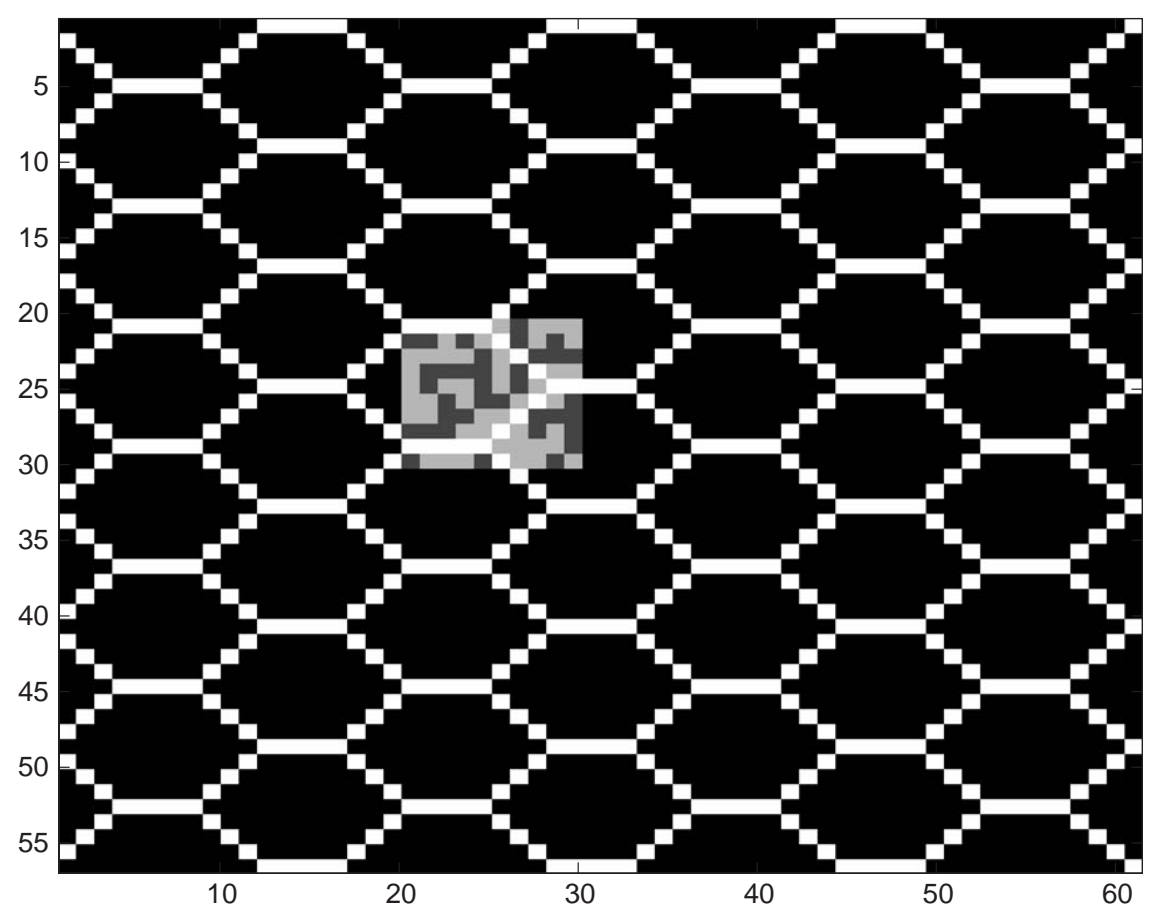

Fig. 5. Initial distribution of the automaton elements. Black spaces are empty, light grey are occupied by cancer cells, dark grey by normal cells, and white by vessels.

The rules of the automaton are inspired by generic features of tumour growth, such as the ability of cancer cells to elude the control (or feedback) mechanisms which maintain stasis in normal tissues. They can also alter their local environment, providing themselves with better conditions for growth and, eventually, for invading the host organism (King, 1996). This phenomenon is implemented in our simulations by allowing the cancer cells to survive under lower local levels of oxygen.

In practice, cancer cells exhibit a remarkable ability to endure very low levels of oxygen. A well-known, but not exclusive, characteristic of cancer cells is their ability under hypoxic conditions to enter a quiescent state, in which they suspend all activity, including any cell division that is not essential for survival (Royds et al., 1998). This ability seems to be related to mutations of the tumour supressor gene $\mathrm{p} 53$. Cells which possess the normal, wild type gene undergo p53-mediated apoptosis under hypoxia (Kinzler and Vogelstein, 1996). By contrast, cells with mutations of the p53 gene can survive hypoxia in a quiescent state (Royds et al., 1998). They can remain in this latent state for a certain period of time, before starving to death. In addition, these cells may express growth factors that stimulate angiogenesis. In our model, we assume that all cancer cells are expressing a mutant form of the $\mathrm{p} 53$ gene, and that this enables them to survive much longer than normal cells under hypoxia. Despite the crudeness of our assumption, we still believe our results will be significant, since at least $50 \%$ of all human tumours possess cells with mutations in p53 (Royds et al., 1998).

Finally, we incorporate into our model competition between cancer and normal cells for the existing resources (nutrients, metabolites, etc.) (Gatenby, 1996). Normal tissue is a non-competitive cell community, since, under conditions such as overcrowding or starvation, feedback mechanisms act to maintain tissue stasis. However, when members of this community become cancerous, a new population, with its own dynamics, is formed. For further progression of this transformed population to occur, the tumour cells must compete for space and resources with the normal cells. Such competition is also introduced in our model.

\subsection{Automaton rules}

The rules governing the evolution of the automaton elements are as follows.

1. An element that is empty or occupied by a vessel does not evolve directly. An empty element can evolve indirectly when cell division takes place in a neighbouring element that is occupied by a cell.

2. The distribution of oxygen is calculated by solving an appropriate boundary value problem, described below.

3. We determine the type of cell in an occupied element from its state vector. Cells attempt to divide at each 
time step. ${ }^{5}$ The rules of the division process depend on the type of cell.

4. For a normal cell, we first determine the local oxygen concentration. If it is below a threshold value, the cell dies and otherwise it attempts to divide. We determine the threshold value by sampling the occupation state of its nearest neighbours. If the cell is surrounded by more normal than cancer cells, then the threshold is fixed at $N_{T 1}$. If there are more cancer cells than normal ones then the threshold is fixed at $N_{T 2}>N_{T 1}$.

5. The rules for cancer cells are similar to those for normal cells, except that the comparison between the oxygen level and the threshold value determines only whether the cell is going to divide: the rules for death of a cancer cell are determined independently (see below). If there are more cancer cells than normal ones surrounding the cell, then its threshold is fixed at $C_{T 1}$, and otherwise it is fixed at $C_{T 2}>C_{T 1}$. We assume that $C_{T 1}<C_{T 2}$ and $N_{T 1}<N_{T 2}$ which means that cells are more likely to divide if they are mainly surrounded by cells of the same type. Mathematically, this introduces competition into our model.

6. Elements occupied by cancer cells whose local oxygen concentration falls below threshold enter a quiescent or latent state, during which most of the cell's functions are suspended, including proliferation. On entering this state, a clock is started. The clock is incremented by one unit for each iteration the cell remains in the quiescent state, i.e. while the oxygen level remains below threshold. If the clock reaches a given value the cell dies. However, if at some time the oxygen level goes above threshold the cell returns to the proliferating state and its clock reset to zero.

7. Elements occupied by normal and cancer cells are sinks of oxygen.

8. If the oxygen level at an element occupied by a cell is above threshold, then we determine whether cell division occurs by sampling in a region of radius $R$ around the element. ${ }^{6}$ If there is only one empty space, then the cell divides, and the new cell occupies this empty space. If there is more than one empty space, the new cell goes to the free element with the largest oxygen concentration (Patel et al., 2001). If there is no empty space, the cell fails to divide, and dies (Kansal et al., 2000).

\subsection{Boundary-value problem for the oxygen distribution}

The next step is to formulate and solve the boundaryvalue problem for the extracellular oxygen concentration.

\footnotetext{
${ }^{5}$ Consequently we are assuming that the duration of the cell-cycle is the same for cancer and normal cells. This is the time-scale we assign to each iteration of our algorithm.

${ }^{6}$ In the simulations below, $R=1$ in element units.
}

To this end, we follow the procedure explained in Patel et al. (2001). The corresponding field is, in principle, timedependent and, therefore, so should be the corresponding partial differential equation (PDE) which determines its spatiotemporal evolution. However, as oxygen molecules are negligible in mass compared to the mass of a typical cell, they reach their equilibrium state on a much shorter time-scale than the cell distribution. This allows us to apply the so-called adiabatic approximation, for which the time evolution of the oxygen concentration is so fast compared to the evolution of the cells that it can be considered instantaneously in steady state. This means that the evolution of the oxygen distribution is solved by successive solution of an elliptic boundary-value equation rather than a parabolic (evolutionary) PDE.

The full PDE we should solve to obtain the distribution of extracellular oxygen, $P(\vec{x}, t)$, is given by

$\frac{\partial P}{\partial t}=D_{P} \nabla^{2} P-k(\vec{x}) P$,

where $D_{P}$ is the oxygen diffusion coefficient, and $k(\vec{x})$ is the oxygen uptake rate at position $\vec{x}$. In the adiabatic approximation Eq. (6) becomes

$D_{P} \nabla^{2} P-k(\vec{x}) P=0$.

The function $k(\vec{x})$, which is updated on each iteration of our automaton, depends on the cell distribution and is defined by

$k(\vec{x})= \begin{cases}k_{N} & \text { if there is a normal cell at } \vec{x}, \\ k_{C} & \text { if there is a cancer cell at } \vec{x}, \\ 0 & \text { otherwise }\end{cases}$

It remains to prescribe appropriate boundary conditions for Eq. (7). This is a crucial point, as the boundary conditions we impose at the vessels will couple the dynamics of the tissue with the distribution of haematocrit in the vascular network. Oxygen enters the system by crossing the walls of the vessels, the flux being given by $\vec{J}=-D_{P} \nabla P$. Thus we impose the following mixed boundary conditions at the walls of the vessels:

$-D_{P} \mathbf{n}_{w} \cdot \nabla P=\mathscr{P}\left(P_{b}-P\right)$,

where $\mathbf{n}_{w}$ is the unit outward vector, orthogonal to the vessel wall, $\mathscr{P}$ is the permeability of the vessel, and $P_{b}$ is the oxygen level inside the vessel, which is essentially the haematocrit. Physically, this means that we are assuming that the rate of leakage of oxygen through the wall of the vessels is equal to its rate of diffusion, meaning that the transport process is in steady state. We also impose no-flux boundary conditions along the edges of our domain, $\Omega$ :

$\left.\mathbf{n}\right|_{\partial \Omega} \cdot \nabla P=0$,

where $\left.\mathbf{n}\right|_{\partial \Omega}$ is the unit outward vector, orthogonal to the boundary of the domain. 
Table 1

Parameter values used in our simulations

\begin{tabular}{|c|c|c|c|}
\hline Parameter & Value & Units & Source \\
\hline$k_{m}$ & 0.83 & $\mathrm{~s}^{-1}$ & Pries et al. (1998) \\
\hline$Q_{\text {ref }}$ & 40 & $\mathrm{nl} \mathrm{min}-1$ & Pries et al. (1998) \\
\hline$k_{s}$ & 1.79 & $\mathrm{~s}^{-1}$ & Pries et al. (1998) \\
\hline$\alpha$ & 0.5 & None & Fung (1993) \\
\hline THR & 2.5 & None & Fung (1993) \\
\hline$D_{P}$ & $2.41 \times 10^{-5}$ & $\mathrm{~cm}^{2} \mathrm{~s}^{-1}$ & Goldman and Popel (2000) \\
\hline$K_{N}$ & $1.57 \times 10^{-4}$ & $\mathrm{ml} \mathrm{O}_{2} \mathrm{ml}^{-1} \mathrm{~s}^{-1}$ & Goldman and Popel (2000) \\
\hline$K_{T}$ & $1.57 \times 10^{-4}$ & $\mathrm{ml} \mathrm{O}_{2} \mathrm{ml}^{-1} \mathrm{~s}^{-1}$ & Estimated \\
\hline $\mathscr{P}$ & $3.0 \times 10^{-4}$ & $\mathrm{~cm} \mathrm{~s}^{-1}$ & Estimated \\
\hline$N_{T 1}$ & $4.5 \times 10^{-4}$ & $\mathrm{~g}$ & Patel et al. (2001) \\
\hline$N_{T 2}$ & $4.5 \times 10^{-3}$ & $\mathrm{~g}$ & \\
\hline$C_{T 1}$ & $1.5 \times 10^{-5}$ & $\mathrm{~g}$ & \\
\hline$C_{T 2}$ & $4.5 \times 10^{-5}$ & $\mathrm{~g}$ & \\
\hline
\end{tabular}

Details on the numerical procedure we use to solve this boundary-value problem are given in Appendix A.

\subsection{Parameter values}

We have managed to find in the literature values for most of the parameters we use in our simulations. Where this has not been possible, we have checked that the results are not sensitive to variations in the corresponding parameter. The only parameters for which we have not found any estimates are $N_{T 2}, C_{T 1}$, and $C_{T 2}$. The values for the other parameters we use are stated in Table 1.

To estimate $\mathscr{P}$ we assume that it scales with the molecular weight, $m$, of oxygen as $\mathscr{P} \sim m^{-1}$. Patel et al. (2001) provide a value of the permeability for glucose, $\mathscr{P}_{G}$, so we estimate the corresponding value for oxygen as $\mathscr{P}_{G} / \mathscr{P}=m_{O_{2}} / m_{G}$.

\subsection{Growth dynamics}

We now apply the procedures described in Sections 3.1 and 3.2, taking as initial condition for the cellular colony the pattern shown in Fig. 5. In order to determine the effect that heterogeneity in the oxygen distribution has on the evolution of the automaton we consider two different oxygen distributions. In the first case the distribution of haematocrit, and hence oxygen, is that obtained from the adaptation mechanism described in Section 2. In the second case we distribute the same amount of oxygen uniformly in our hexagonal network of vessels.

Fig. 6 shows how the total number of cancer cells and the number of quiescent cancer cells evolve (in units of the characteristic time step assigned to cellular division) when the colony is located in a heterogeneous environment. We note that after about 60 time steps both variables saturate to constant values. The population of normal cells falls to zero after a few iterations (results

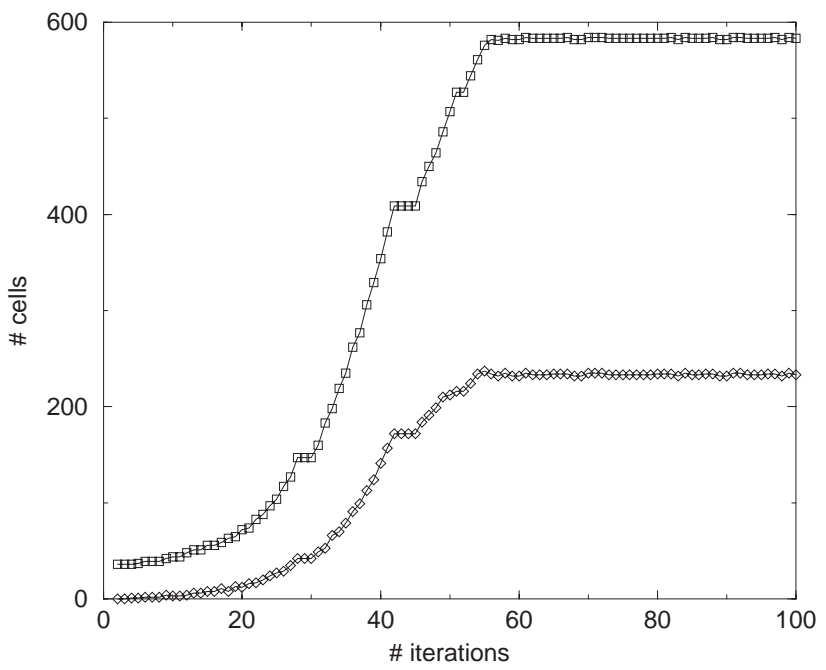

Fig. 6. Graph showing how the total number of cancer cells (squares) and the number of quiescent cancer cells (diamonds) evolve over time when the colony grows in an inhomogeneous environment.

not shown). Similar, qualitative behaviour is observed when we vary the parameters for which we do not have experimental estimates.

More can be learnt about this behaviour by looking at the growth dynamics (see Fig. 7) and the oxygen distribution, (see Fig. 8). As Fig. 7 shows, colony growth is not isotropic, i.e. not at the same rate in all directions, as one would expect in homogeneous conditions. Instead, the colony grows into regions where the oxygen concentration is higher, as a consequence of rule 8 of our automaton. To explain why the colony reaches a finite, maximal size and the population is confined in a given region of the simulation domain we refer back to the automaton rules. If a cell divides and the new cell is situated in a poorly oxygenated region, this cell dies in a few iterations of our automaton. Consequently all viable cells are located in well-oxygenated regions of the domain. While the regions in which the cells are accumulating are rich in oxygen, the amount of oxygen being supplied is finite and can only sustain a certain number of cells. The combination of these two effects leads to the stationary pattern observed in Fig. 7. We have performed simulations in domains with different sizes and consistently observed that the colony size saturates to a value smaller than the size of the domain.

We repeated the simulation described above, evolving the same initial colony under the same automaton rules, but using a uniform oxygen distribution. In order to simulate a homogeneous oxygen environment we distributed the same amount of oxygen into each vessel of our hexagonal network, under the constraint that the total amount of oxygen is the same as in the previous simulation. Referring to Fig. 10 we observe that the growth process is, now, approximately isotropic and 

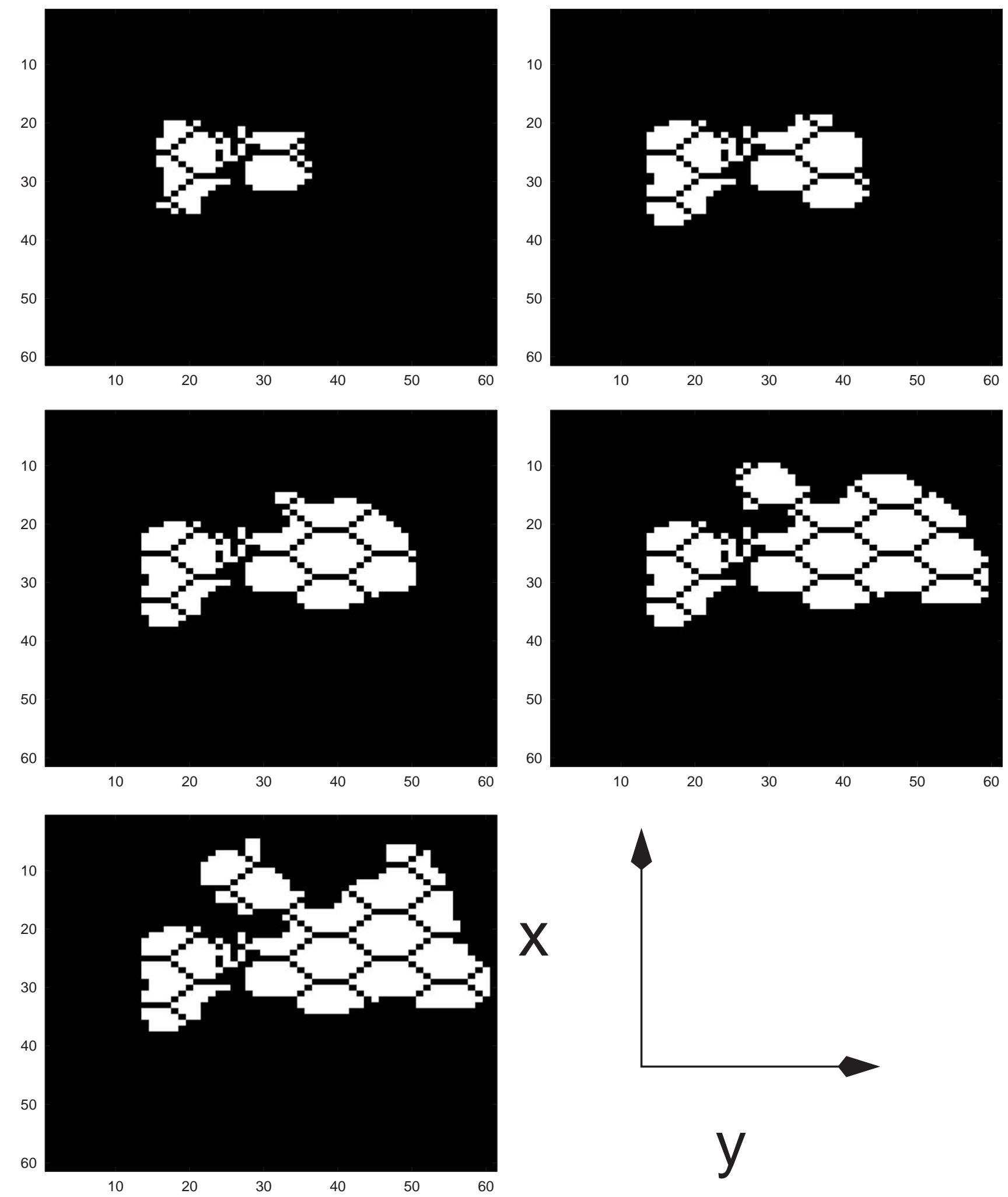

Fig. 7. Series of images showing the growth pattern of our automaton under inhomogeneous conditions after 10, 20, 30, 40, and 60 iterations. White spaces correspond to cancer cells and black spaces to either empty spaces or vessels.

much quicker than in the inhomogeneous case. However, the saturation behaviour shown in Fig. 9 is a finite size effect. (Fig. 10). In other words, unlike the behaviour for colony growth in an inhomogeneous environment, our automaton invades all of the space available. Again, we can understand this behaviour in 


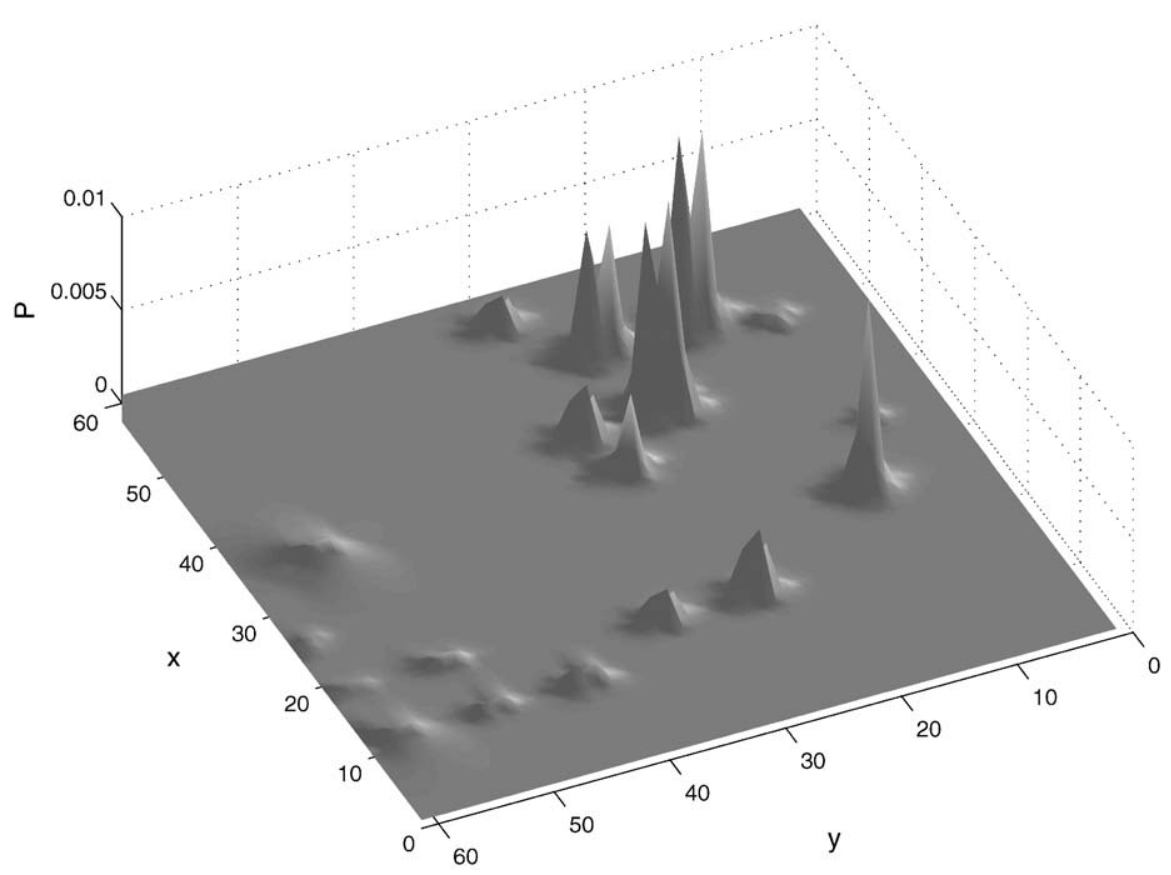

Fig. 8. Graph showing the oxygen distribution after a colony, grown under inhomogeneous conditions, has reached its steady state.

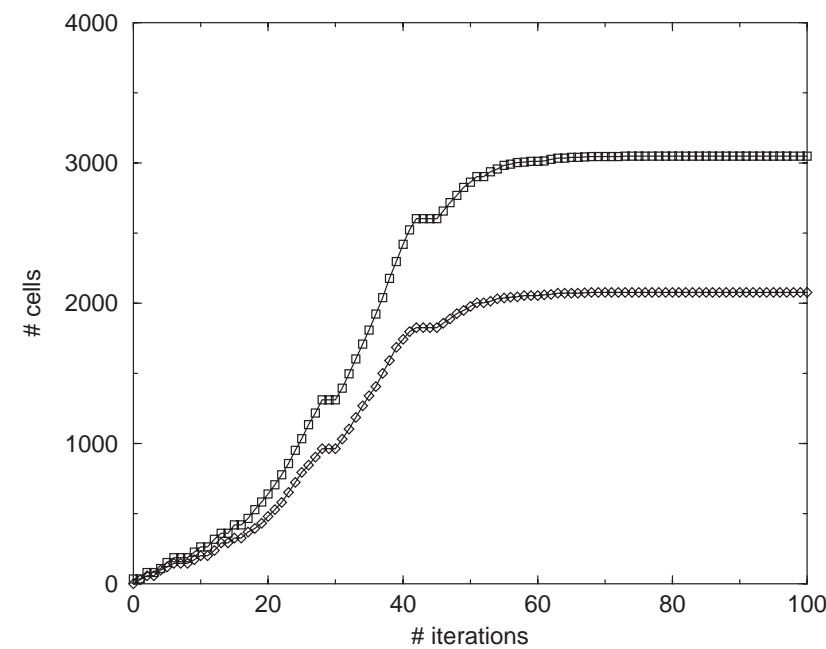

Fig. 9. Graph showing how the total number of cancer cells (squares) and the number of quiescent cancer cells (diamonds) evolve over time when the colony grows in a homogeneous environment.

terms of the distribution of oxygen. Fig. 11 shows that there are now no poorly oxygenated regions, so the colony is free to grow isotropically, until it occupies all the space available.

Comparing Figs. 7 and 9, we conclude that, while a colony of cells may reach a stationary maximum size in an inhomogeneous environment, due to the existence of poorly oxygenated areas, the same colony will invade the entire domain when grown under homogeneous conditions. We remark that the colony size at which growth under homogeneous conditions saturates is much bigger than the corresponding value under inhomogeneous conditions.

To check the robustness of our results to changes in the (unknown) parameters, we have run several simulations taking different values for the parameters $C_{T 1}$ and $C_{T 2}$. The results show that we obtain the same, qualitative behaviour when these parameters vary over three orders of magnitude. From the quantitative point of view, we find that the smaller these parameters are the bigger is the size of the colony at equilibrium as would be expected.

\section{Invasion dynamics}

The second process we study is the malignant invasion of a healthy tissue. We use the same automaton rules as in Section 3 and change only the initial conditions. In this case we distribute normal cells and empty elements randomly in the free space between the vasculature, and place a colony of cancer cells in the centre of the simulation space (see Fig. 12). As in Section 3, we compare the patterns of invasion under two different conditions: homogeneous and inhomogeneous oxygen distribution.

Figs. 13 and 14 show how the number and spatial distribution of the different cell types evolve over time. From Fig. 14 we observe that invasion is greatly hindered by the inhomogeneous distribution of oxygen: this impedes the spread of cells through the space available, as cells that enter poorly oxygenated regions die in a small number of generations (see Fig. 15). This is the same effect that limited colony growth in the previous section. 

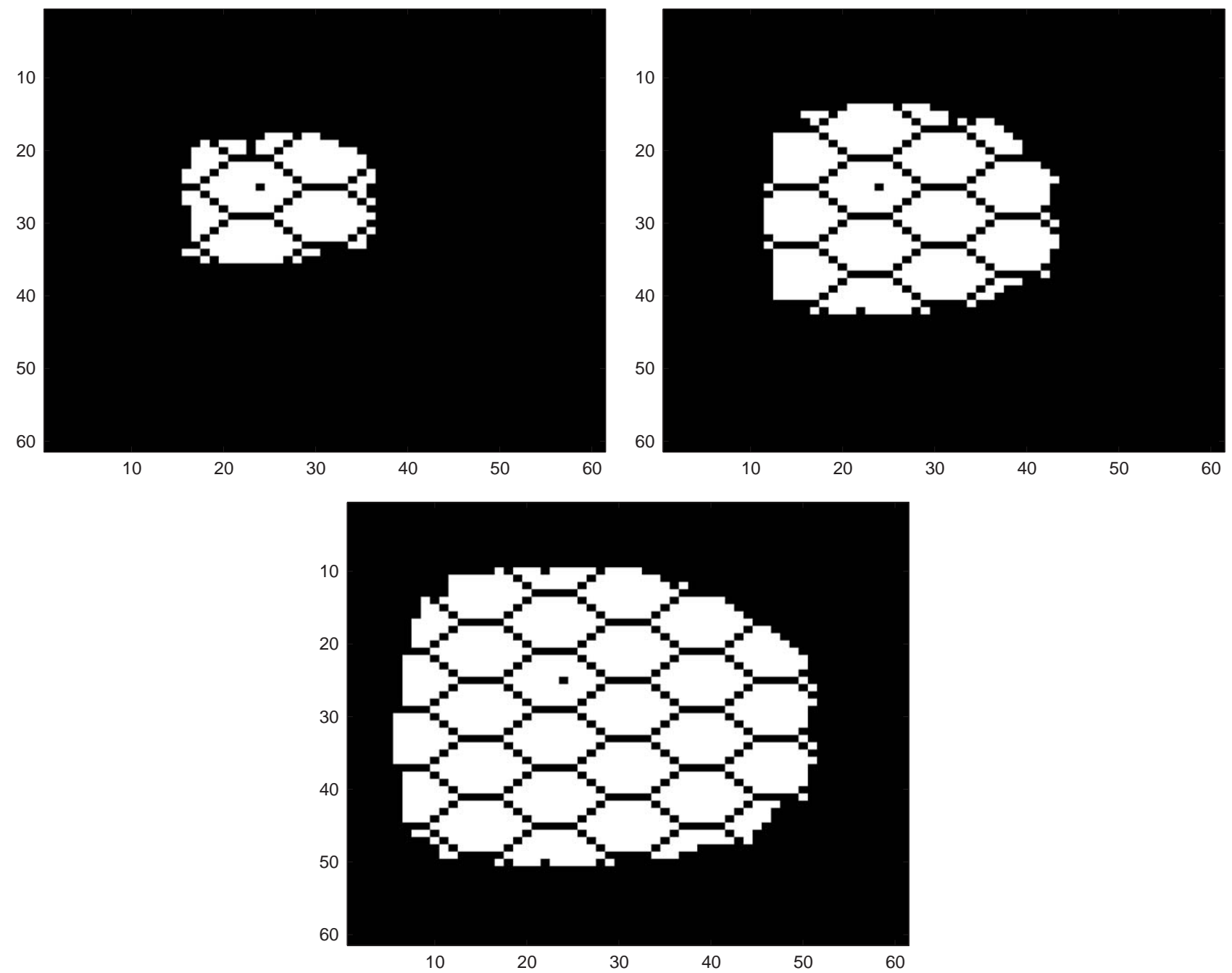

Fig. 10. Series of images showing the growth pattern of our automaton under homogeneous conditions after 10, 20 and 30 iterations.

By contrast, our model predicts almost complete invasion of a homogeneous environment (see Figs. 16 and 17). Again, the growth pattern of the malignant core of cells is roughly isotropic, eventually spreading over the whole tissue, and killing almost all of the normal cells. Such widespread invasion is possibly due to the homogeneity of the oxygen distribution which provides sufficient nutrients for the cancer cells to take over the whole tissue. As in Section 3, the growth saturation of the population is probably a finite size effect. However we believe that the saturation observed in the inhomogeneous case is a genuine feature, arising from the evolution rules in our automaton model.

\section{Discussion}

We have used a simple cellular automaton, whose rules capture some generic features of tumour development, to study the influence of environmental conditions on the evolution of a tissue containing normal and cancerous cells.

Our most important result is that environmental inhomogeneity restricts dramatically the ability of malignant colonies to grow and invade healthy tissue. This is because non-uniform oxygen perfusion leads to the existence of very poorly oxygenated regions which the cancer cells fail to populate: any cells that reach these regions starve after a few number of iterations. By contrast, the same automaton rules yields full invasion of the tissue if it is uniformly perfused. In this case, the malignant core does not find any regions with low levels of oxygen and it grows until, eventually, it occupies all the space available.

Therefore, we predict that one way to combat cancer invasion would be to increase blood flow heterogeneity. While it is not clear that such a strategy would totally eradicate the tumour, it would certainly diminish the supply of drugs to the remainder of the cancer. Indeed, non-uniform blood flow is known to be one of the barriers to drug delivery (Jain, 2001). On the other hand, 




Fig. 11. Oxygen distribution for a colony grown under homogeneous conditions.

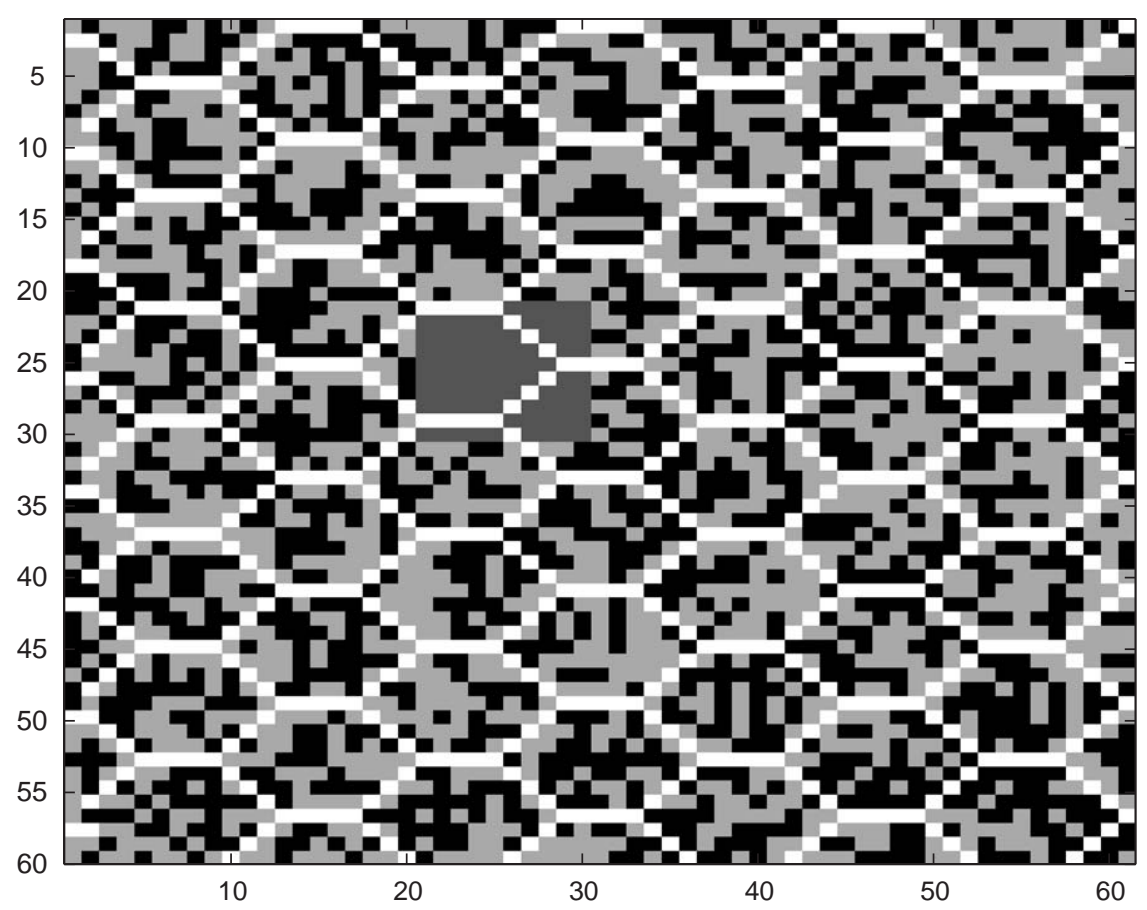

Fig. 12. Initial conditions for simulating invasion. Black spaces are empty, dark grey are occupied by cancer cells, light grey by normal cells, and white by vessels.

the remaining tumour cells would be concentrated in well-oxygenated areas, where they would be more responsive to radiotherapy. In fact, hypoxia is viewed by many investigators as a major contributing factor radiotherapy failure. Consequently some protocols involve hyperoxygenation in attempt to eliminate or lessen the effects of hypoxia prior to or during fractionated radiotherapy. However, this approach is presently rather ad hoc. A more quantitative approach would allow us to refine the model and make it more realistic.

In our cellular automaton model, we have implemented some of the features of tumour growth by means of 


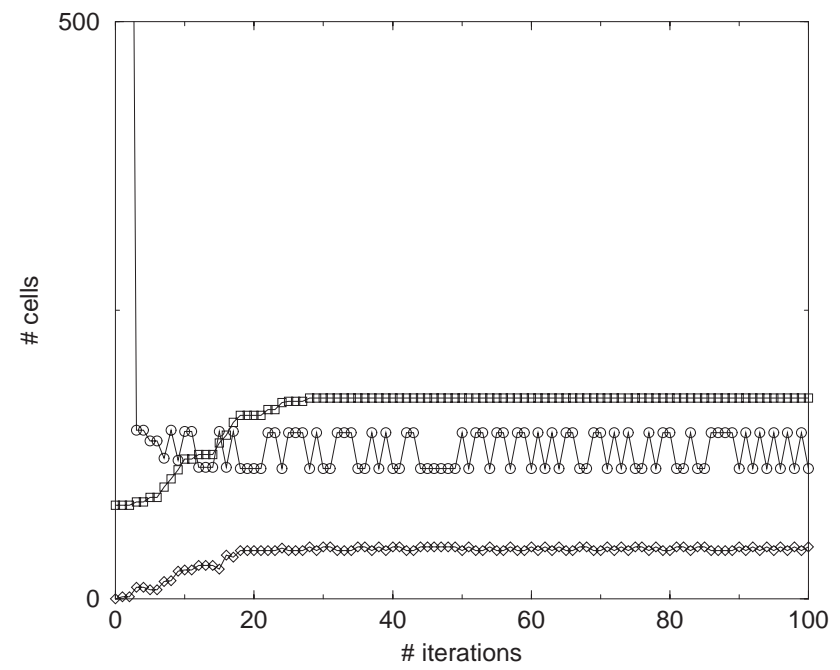

Fig. 13. Number of normal cells (circles), cancer cells (squares), and cancer cells in quiescent state (diamonds) as a function of time for the invasion process in an inhomogeneous environment. suitable rules. Yet, the same biological characteristics could be implemented by means of different rules. Consider, for example, competition. As has already been mentioned, cancer and normal cells compete for the available resources. This has been incorporated in our model by making the "death rule" for a cell depend on the cells that occupy its neighbouring elements. However qualitatively similar effects could have been realized by employing different oxygen consumption rates.

Another weakness of the present model is the fact that the vasculature is independent of the dynamics of the surrounding tissue. This situation is not entirely realistic, for it is known that vasculature adapts to the needs of the tissue (Pries et al., 1998). In order to couple the dynamics of the tissue with the structural adaptation of the vessels we could introduce growth factors into the model. The growth factors would be secreted by environmentally stressed cells and then diffuse through the tissue, modifying the parameters in Eq. (1) when
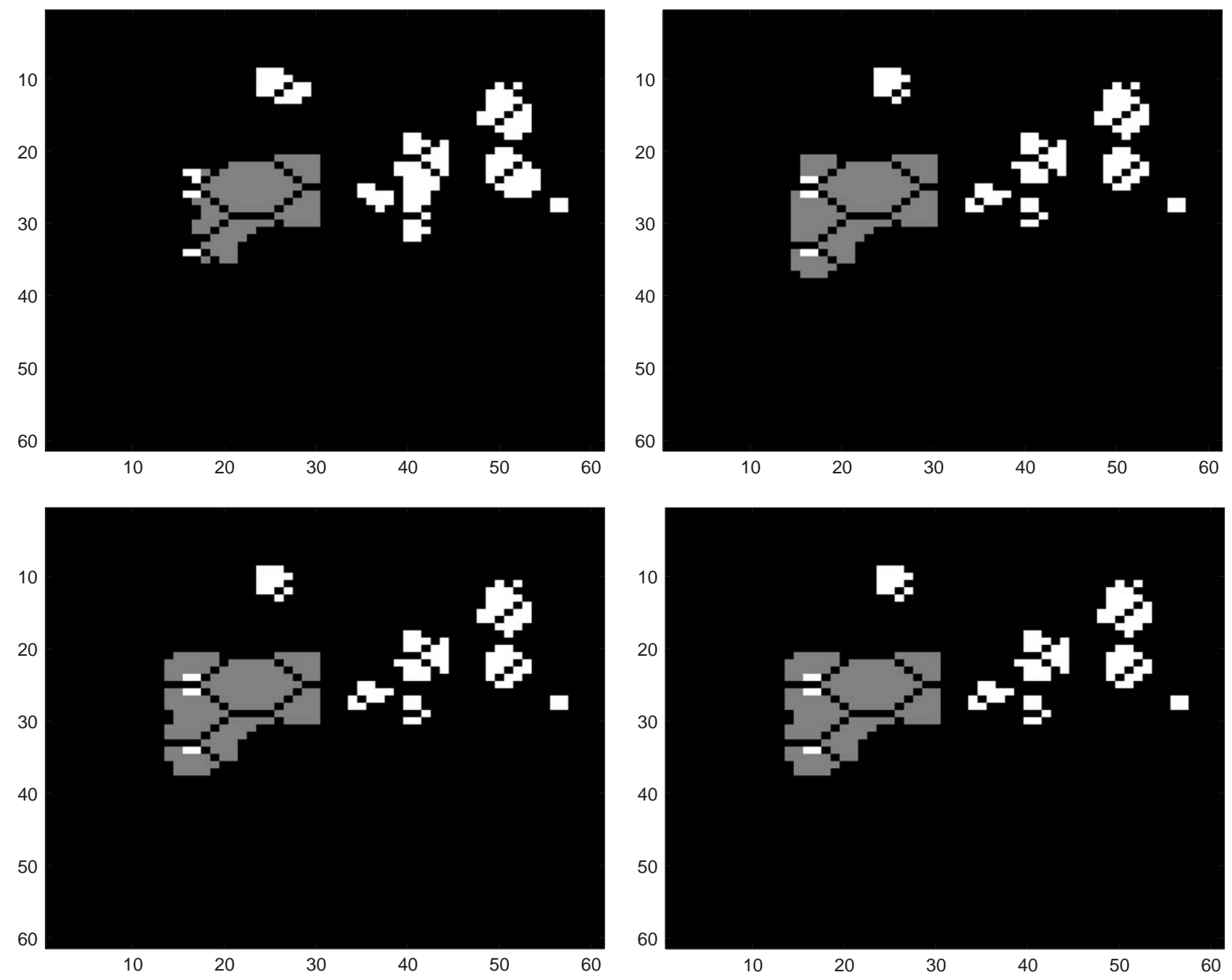

Fig. 14. Series of images showing the pattern of invasion of our automaton under inhomogeneous conditions after 10, 20, 30 and 40 iterations. Grey cells correspond to cancer cells, white to normal cells and black to either empty spaces or vessels. 


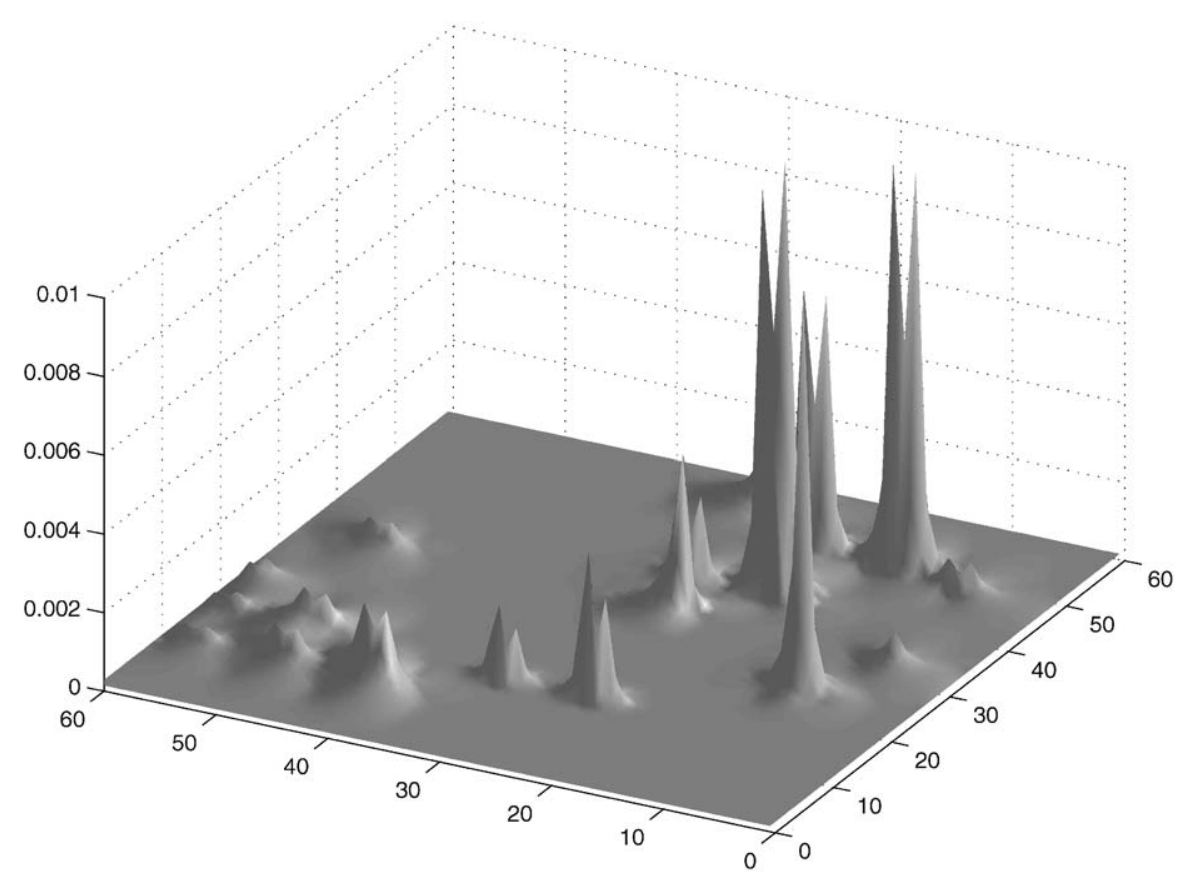

Fig. 15. Distribution of oxygen for a colony grown to steady state under inhomogeneous conditions.

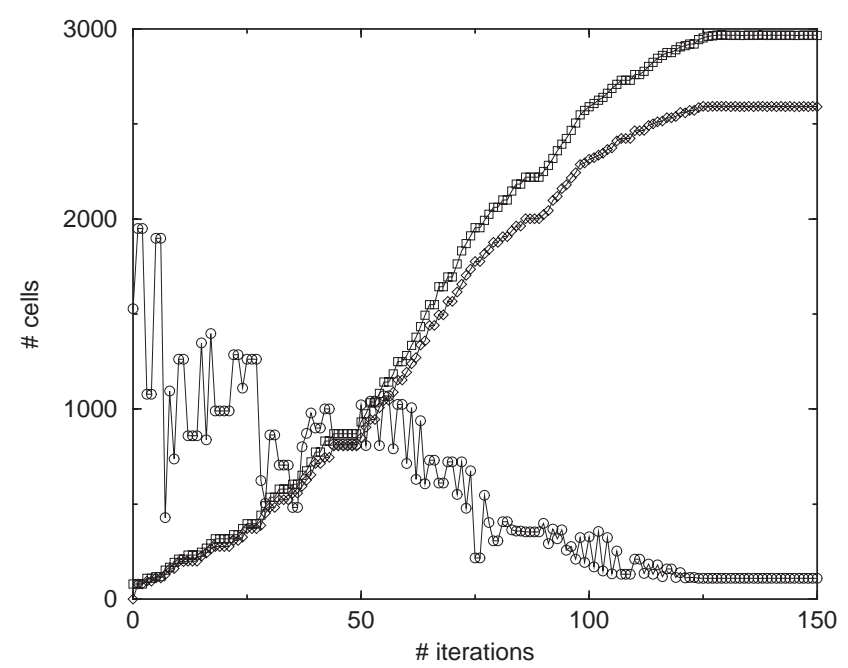

Fig. 16. Number of normal cells (circles), cancer cells (squares), and cancer cells in quiescent state (diamonds) as a function of time for the invasion process in an homogeneous environment.

they reach a vessel. Further model extensions could include incorporating a more detailed description of how the tumour perturbs vascularity and blood flow, in particular, tumour-induced vascular degradation, temporal variations in flow due to transient spasm and thrombosis, and tumour-induced angiogenesis. Once we have coupled tissue dynamics and evolution of the vasculature we can use our model to test the action of different anti-angiogenic drugs. This coupling could be achieved by incorporating into our model the now welldefined hypoxia-mediated HIF1 $\alpha$-VEGF pathway.

Another weakness of our model is the assumption that all cells have the same period of division, and therefore that the cell cycles for normal and cancer cells are identical. We are currently introducing a more accurate description of the process of cell division into our model.

The aim of the present paper is to introduce a modelling framwork which can then be further developed to include the aforementioned issues. Despite the above weaknesses, we can conclude from our model simulations that non-uniformity in nutrient supply caused by blood flow heterogeneity seems to have a strong influence on the patterns of growth and invasion. Consequently it should not be neglected in any serious effort to produce realistic models of tumour growth in vivo.

\section{Acknowledgements}

TA thanks the EU Research Training Network (5th Framework): "Using mathematical modelling and computer simulation to improve cancer therapy" for funding this research. HMB thanks the EPSRC for funding as an Advanced Research Fellow. Part of this work was carried out during a Royal Society Leverhulme Trust Senior Research Fellowship (PKM). The authors thank Mark Chaplain for careful reading of an early version of this manuscript. 

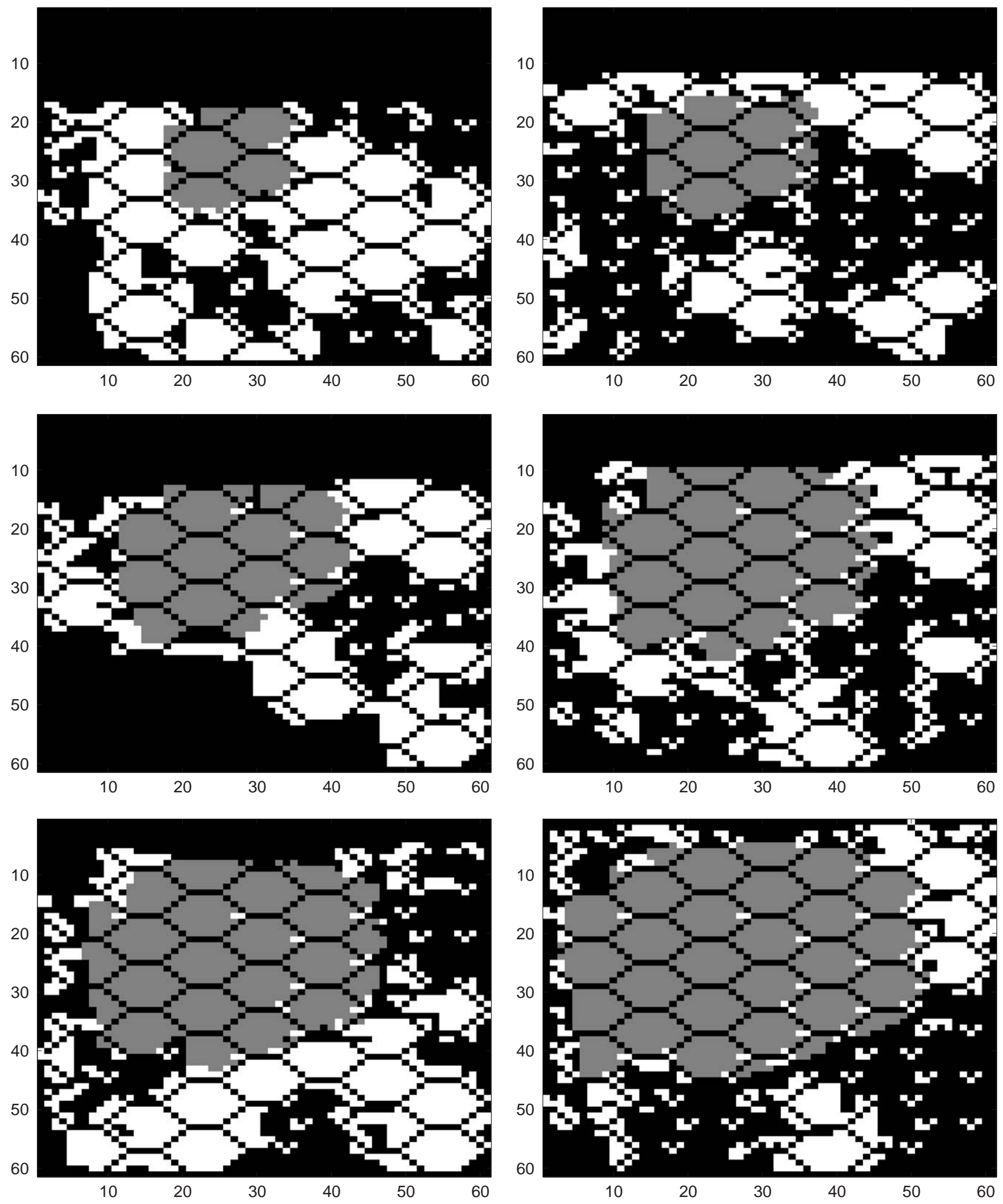

Fig. 17. Series of images showing the pattern of invasion of our automaton under homogeneous conditions after 10, 20, 30, 40, 50 and 60 iterations. Grey cells correspond to cancer cells, white to normal cells and black to either empty spaces or vessels. 


\section{Appendix A. Numerical solution of the boundary-value problem}

Once the boundary-value problem for the distribution of oxygen has been formulated, we proceed to its numerical solution by means of a suitable finitedifference scheme, which basically reduces the boundary value problem to the solution of a large number of (linear) algebraic equations. To begin with, we write Eq. (8) in discrete finite-difference form (Smith, 1985):

$$
\begin{aligned}
& \frac{D_{P}}{\Delta^{2}}\left(P_{j+1, l}+P_{j-1, l}+P_{j, l+1}+P_{j, l-1}-4 P_{j, l}\right) \\
& \quad-k_{j, l} P_{j, l}=0,
\end{aligned}
$$

where the subscripts $j, l$ are the row and column indices, respectively, of the corresponding automaton element. The PDE (Eq. (8)) and the boundary conditions (Eqs. (9) and (10)) must be satisfied simultaneously, so we have to discretise them and introduce the latter into the finite-difference scheme (A.1) when appropriate. Eqs. (9) and (10) in finite-difference form read,

$$
-\frac{D_{P}}{\Delta}\left(P_{j, l}-P_{j, l-1}\right)=\mathscr{P}\left(P_{b}-P_{j, l}\right) \text {, }
$$

where we have assumed that there is a vessel element at $(j, l-1)$, and

$$
\begin{aligned}
& P_{j, L}-P_{j, L+1}=0, \\
& P_{j, 0}-P_{j,-1}=0, \\
& P_{J, l}-P_{J+1, j}=0, \\
& P_{0, l}-P_{-1, l}=0,
\end{aligned}
$$

where $J$ and $L$ are, respectively, the number of rows and columns in our simulation spaces. Hence, if an element $(j, l)$ is one of the neighbours of an element occupied by a vessel at, for instance, $(j, l-1)$, then, by substitution of Eq. (A.2) into Eq. (A.1), the corresponding finitedifference equation becomes

$$
\begin{aligned}
& P_{j+1, l}+P_{j-1, l}+P_{j, l+1}-\left(3+\frac{\Delta^{2} k_{j, l}-\mathscr{P} \Delta}{D_{P}}\right) P_{j, l} \\
& \quad=\frac{\mathscr{P} \Delta}{D_{P}} P_{b} .
\end{aligned}
$$

Similarly, if the element under consideration is on one of the boundaries of the domain, for example $(j, L)$, then from Eqs. (A.1) and (A.3), we have

$P_{j+1, L}+P_{j-1, L}+P_{j, L-1}-\left(3+\frac{\Delta^{2} k_{j, L}}{D_{P}}\right) P_{j, L}=0$.

Eqs. (A.1), (A.4), and (A.5) form a set of linear equations for the level of oxygen at the $(j-l)$ th automaton element, which can be expressed in matrix notation by defining the index $i=J(j-1)+l$, where $j=1, \ldots, J, l=1, \ldots, L$, and $i=1, \ldots, J \times L$, which implies that the size of the set of equations we must solve is $J \times L$. In our present simulations, $J=L=61$.
Due to the mixed boundary conditions at the walls of the vessels, the corresponding matrix is a non-symmetric band diagonal sparse matrix (Press et al., 1992).

There is a variety of methods for solving linear systems with a very large number of algebraic equations. Most of them exploit the fact that the corresponding matrix is sparse. We choose one of the simplest ones, which is applicable in the case of band diagonal matrices (Press et al., 1992). Generally speaking, band diagonal matrices have $m_{1}$ non-zero elements immediately below the diagonal and $m_{2}$ non-zero elements immediately above it. If $m_{1}, m_{2} \ll N$, i.e. the matrix is sparse, then the linear system can be solved using $L U$ decomposition, even when $N \gg 1$. The key step in this method is storing the matrix in the so-called compact form. This form results from tilting the matrix $45^{\circ}$ clockwise, so that its non-zero elements lie in a long, narrow matrix with $m_{1}+m_{2}+1$ columns and $N$ rows. This introduces a dramatic decrease in the dimension of the matrix, and, consequently, in the number of operations needed to solve the system of equations. Unfortunately, it is not possible to store the $L U$ decomposition of a band diagonal matrix as compactly as the matrix itself, as the decomposition, basically carried out by Crout's method, produces additional non-zero elements which need to be allocated. A straightforward storage scheme (Press et al., 1992 ) is to return the upper triangular factor, $U$, in the same space that the compact form of the matrix used to occupy, and to return the lower triangular factor to an additional $N \times m_{1}$ matrix. We have checked our numerical routine for smaller systems with standard (i.e. non-compact) $L U$ decomposition, and found the agreement between them to be excellent.

\section{References}

Alarcón, T., Byrne, H.M., Maini, P.K., 2002. Design principle of vascular beds: effects of blood rheology and transmural pressure, submitted.

Anderson, A.R.A., Chaplain, M.A.J., 1998. Continuous and discrete mathematical models of tumor-induced angiogenesis. Bull. Math. Biol. 60, 857-899.

Baish, J.W., Gazit, Y., Berk, D.A., Nozue, M., Baxter, L.T., Jain, R.K., 1996. Role of tumour architecture in nutrient and drug delivery: an invasion percolation-based model. Microvasc. Res. 51, 327-346.

Baum, M., Chaplain, M.A.J., Anderson, A.R.A., Douek, M., Vaidya, J.S., 1999. Does breast cancer exist in a state of chaos? Eur. J. Cancer 35, 886-891.

Duchting, W., Vogelsaenger, T., 1985. Recent progress in modelling and simulation of three dimensional tumour growth and therapy. Biosystems 18, 79-91.

Ermentrout, G.B., Edelstein-Keshet, L., 1993. Cellular automata approaches to biological modelling. J. Theor. Biol. 160, 97-133.

Fung, Y.C., 1993. Biomechanics. Springer, New York.

Gatenby, R.A., 1996. Application of competition theory to tumour growth: implications for tumour biology and treatment. Eur. J. Cancer 32A, 722-726. 
Goldman, D., Popel, A.S., 2000. A computational study of the effect of capillary network anastomoses and tortuosity on oxygen transport. J. Theor. Biol. 206, 181-194.

Honda, H., Yoshizato, K., 1997. Formation of the branching pattern of blood vessels in the wall of the avian yolk sac studied by computer simulation. Dev. Growth Differentiation 39, 581-589.

Jain, R.K., 2001. Delivery of molecular and cellular medicine to solid tumours. Adv. Drug Delivery Rev. 46, 149-168.

Kansal, A.R., Torquato, S., Harsh IV, G.R., Chiocca, E.A., Deisboeck, T.S., 2000. Simulated brain tumour growth dynamics using a threedimensional cellular automaton. J. Theor. Biol. 203, 367-382.

King, R.J.B., 1996. Cancer Biology. Longman, Harlow.

Kinzler, K.W., Vogelstein, B., 1996. Life (and death) in a malignant tumour. Nature 379, 19-20.

LaBarbera, M., 1990. Principles of design of fluid transport systems in zoology. Science 249, 992-1000.

McDougall, S.R., Anderson, A.R.A., Chaplain, M.A.J., Sherratt, J.A., 2002. Mathematical modelling of flow through vascular networks: implications for tumour-induced angiogenesis and chemotherapy strategies. Bull. Math. Biol. 64, 673-702.

Murray, C.D., 1926. The physiological principle of minimum work, I: the vascular system and the cost of blood volume. Proc. Natl Acad. Sci. USA 12, 207-214.

Patel, A.A., Gawlinsky, E.T., Lemieux, S.K., Gatenby, R.A., 2001. Cellular automaton model of early tumour growth and invasion: the effects of native tissue vascularity and increased anaerobic tumour metabolism. J. Theor. Biol. 213, 315-331.

Press, W.H., Teukolsky, S.A., Vetterling, W.T., Flannery, B.P., 1992. Numerical Recipes in C. Cambridge University Press, Cambridge, UK.

Pries, A.R., Secomb, T.W., Gessner, T., Sperandio, M.B., Gross, J.F., Gaehtgens, P., 1994. Resistance to blood flow in microvessels in vivo. Circ. Res. 75, 904-915.

Pries, A.R., Secomb, T.W., Gaehtgens, P., 1995. Design principles of vascular beds. Circ. Res. 77, 1017-1023.

Pries, A.R., Secomb, T.W., Gaehtgens, P., 1998. Structural adaptation and stability of microvascular networks: theory and simulations. Am. J. Physiol. 275, H349-H360.

Qi, A.S., Zheng, X., Du, C.Y., An, B.S., 1993. A cellular automaton model of cancerous growth. J. Theor. Biol. 161, 1-12.

Royds, J.A., Dower, S.K., Qwarstrom, E.E., Lewis, C.E., 1998. Response of tumour cells to hypoxia: role of p53 and NFkB J. Clin. Path: Mol. Pathol. 51, 55-61.

Smith, G.D., 1985. Numerical Solution of Partial Differential Equations: Finite Difference Methods, 3rd Edition. Oxford University Press, Oxford, UK.

Zamir, M., 1977. Shear forces and blood vessel radii in the cardiovascular system. J. Gen. Physiol. 69, 449-461.

Wolfram, S., 1986. Theory and Applications of Cellular Automata. World Scientific, Singapore. 